\title{
Knowledge on the National programme on Elimination of Mother-to- Child Transmission (EMTCT) of HIV and Syphilis among Primary Healthcare Workers in the district of Kalutara
}

\author{
W. C. J. K. Jayakody, V. T. Silva, N. S. A. Pamila, T. P. Weerasooriya
}

\begin{abstract}
Introduction: The Elimination of Mother-to-Child Transmission (MTCT) of HIV and syphilis infections can be addressed with similar strategies. The availability of testing and more efficacious drug regimens for HIV and syphilis provides an unprecedented global health opportunity to achieve the elimination goals. Currently, Sri Lanka is implementing a National Programme for the EMTCT of syphilis and HIV Island wide. It is important to know the knowledge, attitude and barriers for the EMTCT programme among primary health care workers to achieve the elimination targets.
\end{abstract}

Method: A descriptive cross sectional study was carried out among 200 primary healthcare workers in 10 Medical Officer of Health (MOH) areas in Kalutara district. Data were collected by using a selfadministered questionnaire and analysed by using SPSS.

Results: The majority (60\%) of the health staff was between $40-60$ years and public health midwives (80\%). Majority (66\%) were not exposed to any awareness programmes on it. Majority $(82.5 \%)$ were well aware about testing and interpretation of the results of screening tests for syphilis and HIV. Most of them knew that paediatric HIV and congenital syphilis are preventable diseases. However, majority did not know that HIV could be transmitted through breast milk. The main barrier of the programme is the lack of manpower for additional workload.

Conclusions: Although the primary health care workers are aware the existence of the national programme of EMTCT of Syphilis and HIV in Sri Lanka, they do not have sufficient understanding about the purpose of the EMTCT programme.

Key words: Syphilis, elimination of mother to child transmission, EMTCT, Kalutara, Sri Lanka

Authors: Corresponding author: Dr W. C. J. K. Jayakody, MBBS, PgD Ven, MD; Consultant Venereologist, STD Clinic, Kalutara, Sri Lanka, Email:wcjksovis@gmail.com

Dr. V. T. Silva, Bsc; Programme \& Planning Assistant, STD Clinic, Kalutara.

Dr. N. S. A. Pamila, MBBS, STD Clinic, Kalutara

Dr. T. P. Weerasooriya, MBBS; STD Clinic, Kalutara,

Acknowledgement: All health staff in $\mathrm{MOH}$ stations in Kalutara district, Dr. Lilani Rajapaksa, Consultant Venereologists,

Dr. Jayadarie Ranatunga, Consultant Venereologist and the Staff of STD clinic, Kalutara

Conflict of interest: No conflict of interest

Funding: No funding support for this study

Originality, previous publications or presentations: This is an original work, not published or presented anywhere.

Submitted: 29.11.2015, Accepted: 15.12.2015 


\section{Full article}

\section{Introduction}

Syphilis and HIV are sexually transmitted infections that can lead to serious adverse pregnancy outcomes which cause substantial newborn morbidity, mortality \& global health burden (1). Nearly all HIV infections due to mother to child transmission can be prevented by wide implementation of primary prevention, use of antiretroviral drugs, safe delivery practices and safe infant feeding practices.

Prevention strategies for HIV and syphilis among newborns are similar and can be easily incorporated in to existing national maternal, newborn and child health programmes. The availability of testing and more efficacious drug regimens for HIV and syphilis provides an unprecedented global health opportunity to achieve the elimination goals. World Health Organization (WHO) has set elimination targets of MTCT of syphilis and HIV as less than 0.5 cases per 1000 live births $(<50 / 100$ 000 live births) for both diseases for dual elimination policies (1). Furthermore, WHO identified Sri Lank as one of the potential countries which can get the qualification status of elimination of MTCT syphilis and HIV.

The prevention programmes of MTCT of syphilis and HIV were started in Sri Lanka in 1952 and 2002 respectively (2). Sri Lanka has been a pioneer in the South Asia region in preventing congenital syphilis. Elimination of congenital syphilis was introduced in 2009 in Sri Lanka (3). New dual elimination strategy for congenital syphilis and paediatric HIV is being currently implemented in Sri Lanka since 2013. This program is a collaborative effort of the Family Health Bureau (which is responsible for maternal and child health) and National STD/AIDS Control Programme.

There is well established, and organized preventive health care infrastructure as Medical Officer of Health (MOH) areas in the country where skilled primary healthcare
(PHC) staff is providing maternal, child and family planning services to the catchment area. The public health staff includes doctors, public health nursing sisters (PHNS) and public health midwives (PHM). This system is well supported by data recording and reporting system with monitoring and evaluation and disease surveillance.

Before launching the dual elimination programme, a strategy document was published with clear guidance and action plans. The programme is regulated and guided through regular reviews by steering committee. Teaching, training, technical guidance and infrastructure facilities were provided to primary health care staff. The programme is funded by the GOSL and is being supported by international funding agencies such as UNICEF.

The existing situation in Sri Lanka is in favour of achieving the elimination goals. But primary healthcare (PHC) staff is over burdened with additional work of the EMTCT programme. Therefore, it is important to ascertain the level of knowledge, attitudes and the barriers on this programme to achieve the elimination targets.

\section{Methods}

A descriptive cross sectional study was carried out in Kalutara district in the Western Province. The study was carried out in Kalutara Medical Officer of Health $(\mathrm{MOH})$ area during June 2015 to August 2015. Owing to limited time interval, $10 \mathrm{MOH}$ areas out of 13 were randomly selected. Sample of 200 Primary Health Care (PHC) staff was taken. The study population was the doctors, public health nursing sisters and public health midwives. The information was collected to assess their knowledge, attitudes and practices on the elimination programme.

Selected $\mathrm{MOH}$ areas were visited during the monthly conference day or their educational day for data collection. A self-administered questionnaire in Sinhala language was given 
to the primary healthcare workers those who are willing to participate for the study. Following the completion of the questionnaire, awareness was done on the EMTCT of syphilis and HIV. Data were analysed using SPSS.

\section{Results}

In the study population, majority were female (91\%) and more than 40 years old (69.5\%) (Table 1) Almost four fifth of them were public health midwives (80\%). Majority were advanced level qualified. Approximately one quarter of them was degree holders. It is significant that more than one third of them were having over 20 years of experience in their respective services.

Table1: Socio-demographic profile of GPs $(\mathrm{N}=\mathbf{2 0 0})$

\begin{tabular}{lrr}
\hline Age & Frequency & $\%$ \\
\hline $20-29$ & 18 & 9 \\
$30-39$ & 43 & 21.5 \\
$40-49$ & 60 & 30 \\
$50-59$ & 60 & 30 \\
$>60$ & 3 & 1.5 \\
Not mentioned & 16 & 8 \\
\hline Sex & Frequency & $\%$ \\
\hline Male & 18 & 9 \\
female & 182 & 91 \\
\hline Educational & Frequency & $\%$ \\
qualifications & & \\
\hline O/L & 19 & 9.5 \\
A/L & 162 & 61 \\
Degree/Dip & 49 & 24.5 \\
Post Graduate & 9 & 3.5 \\
Not mentioned & 3 & 1.5 \\
\hline Service duration & Frequency & $\%$ \\
\hline$<5$ & 25 & 12.5 \\
$6-10$ & 35 & 17.5 \\
$11-15$ & 35 & 17.5 \\
$16-20$ & 21 & 10.5 \\
$>21$ & 74 & 37 \\
Not mentioned & 10 & 5 \\
\hline & &
\end{tabular}

Although most (64\%) of them were aware that congenital syphilis is a treatable and preventable condition, majority $(52 \%)$ did not know that babies of congenital syphilis could be asymptomatic.

It is noteworthy that majority (55\%) did not know that HIV could be transmitted through breast milk.

Majority (82.5\%) were well aware about testing and interpretation of the results of screening tests for syphilis and HIV.

Almost all (98.5\%) the primary healthcare staff knew that pregnant mothers need to be screened for HIV and syphilis before 12 weeks. Majority (74\%) did not know that delay in transport of blood sample might affect the results.

Although most of them (89\%) have heard of the programme, only one third (34\%) had participated for the meeting or conference related to this topic. Unfortunately, their awareness on publication on strategies for elimination programme and the health circular relevant to it was poor.

\section{Stigma, discrimination and confidentiality issues}

Almost $90 \%$ of the study population knew that children with HIV and syphilis need not be separated from the normal children in the clinic. Majority wanted to maintain the confidentiality of the pregnant women with HIV but not for syphilis although both are sexually transmitted diseases. Almost all (99\%) knew the importance of the programme and tried to take the blood sample of the pregnant mothers before 12 weeks.

Over three quarters (83.5\%) of study population were very conscious on not to discriminate the patients with STls but majority (86.5\%) did not know that they could not reveal the mother's disease status to their partners.

The main barrier of the programme is the lack of manpower for additional workload. PHC 
staff also highlighted the difficulty in getting a sample of blood since there were no trained personnel to draw blood in every antenatal clinic setup. Another drawback for the testing was that some mothers have already been tested in private sector on the request of Visiting Obstetricians \& Gynaecologists (VOGs). Limited information has been gone to primary health staff about documentation on positive and negative results on ANC records.

\section{Discussion}

This study provided information on primary health care workers understanding and knowledge on the dual elimination programme. This was a representative sample of primary healthcare workers who were engaging in public health activities. In our study sample, majority were females, advance level qualified and over 40 years old. Most primary healthcare workers were having over 20 years of experience of their service and they were well known of antenatal care services and their responsibilities.

Our findings indicated that they had positive attitudes on the programme, but few shortcomings were due to lack of proper understanding of the programme. Their awareness on the latest strategies on the dual elimination was not adequate to make the programme more successful. Majority did not know about the general circular issued by the Ministry of Health on the EMTCT of syphilis and HIV under the number of 02-02/2014.

Further, our findings indicated that majority had adequate knowledge of on paediatric HIV and congenital syphilis. But it is high time to update their knowledge to increase the maximum case detection. Their knowledge on screening tests for two conditions was satisfactory. Almost all knew that pregnant mothers need to be screened for HIV and syphilis before 12 weeks. They had a sound understanding on screening and confirmatory tests of HIV and syphilis.

The stigma, discrimination and the confidentiality issues related to STIs were the major drawbacks identified by the study. The important issue needed to be addressed was that the confidentiality should be maintained for all the sexually transmitted infections and not only for HIV.

The program is being launched island wide and our elimination targets need to be achieved by 2017 (4). It should be noted that thorough and in detail awareness on the programme might support to achieve the target.

\section{Conclusions}

The primary healthcare workers were aware about the existence of the National programme of EMTCT of Syphilis and HIV in Sri Lanka, but they did not have sufficient understanding about the purpose of the EMTCT programme. Their attitude on the programme was encouraging. The public health staff should be made aware on available guidelines and health circulars by the regional authorities. A proper system which is emphasized in EMTCT training programmes need to be established for storage and transportation of blood sample from antenatal clinic to STD clinic. Health manpower needs to be improved quantitatively and qualitatively to achieve EMTCT targets.

\section{References}

1. World Health Organization. Global Guidance on Criteria and Processes for Validation: Elimination of Mother-ti-child Transmission of HIV and Syphilis Geneva: WHO; 2014.

2. National STD/AIDS Control Programme. Guideline for the Management of Maternal Syphilis and Congenital Syphilis Colombo: NSACP; 2011.

3. National STD/AIDS Control Programme. Strategy for the elimination of congenital syphilis in Sri Lanka Colombo: NSACP; 2009.

4. World Health Organization. Investment case for eliminating mother-to-child transmission of syphilis Geneva: WHO Press; 2012. 